\title{
Estudo da doença de Gaucher em Santa Catarina
}

\section{Study of Gaucher disease in Santa Catarina}

Jovino S. Ferreira ${ }^{1}$

Vera Lúcia P. C. Ferreira ${ }^{2}$

David C. Ferreira ${ }^{3}$

\begin{abstract}
A doença de Gaucher (DG) foi a primeira doença de armazenamento lisossomal descrita e a mais encontrada. Caracteriza-se pela deficiência hereditária da atividade da enzima lisossomal glucocerebrosidase, que bloqueia o metabolismo do glicocerebrosídeo. A proposta deste trabalho foi estudar as características clínicas, laboratoriais e radiológicas, as principais mutações encontradas, relacionando-as com as formas clínicas e avaliar a resposta à terapia de reposição enzimática (TRE) nos pacientes com DG em Santa Catarina. Foram estudados dez pacientes com DG no Hospital Universitário, no período entre 1998 e 2003, após confirmação diagnóstica da doença pela dosagem da enzima beta-glicosidase em leucócitos. Pesquisa das mutações foi realizada em amostras de sangue e de mucosa oral. A média de idade ao diagnóstico foi de 19,6 anos. A DG tipo 1 foi diagnosticada em $80 \%$ dos casos, e a tipo 2 em 20\%. Quatro pacientes tiveram história familiar de DG. Hepatoesplenomegalia foi a manifestação clínica mais comum. Anemia e trombocitopenia ocorreram em $100 \%$ dos casos. Dores ósseas foram relatadas por $75 \%$ dos pacientes. Os alelos mutantes encontrados foram N370S e L444P. Houve elevação dos níveis de hemoglobina em todos os pacientes com DG tipo 1. Concluímos que a DG tipo 1 é a forma clínica mais comum. Anemia, trombocitopenia, hepatoesplenomegalia e osteopenia são as características mais freqüentes dos pacientes com DG. O alelo N370S é o mais freqüente, estando relacionado com o tipo 1. O alelo L444P em homozigose sugere letalidade precoce. A TRE é segura e efetiva para a DG tipo 1. Rev. bras. hematol. hemoter. 2008;30(1):5-11.
\end{abstract}

Palavras-chave: Doença de Gaucher; lipidoses; glucocerebrosidase; genótipo.

\section{Introdução}

A doença de Gaucher (DG) foi a primeira doença de armazenamento lisossomal descrita e é a lipidose mais freqüentemente encontrada. É caracterizada pela deficiência hereditária da atividade da enzima lisossomal glucocerebrosidase (ou beta-glicosidase), que hidrolisa o glicocerebrosídeo glucosilceramida em glicose e ceramida. Tal deficiência induz ao acúmulo deste glicolipídio, levando às altera- ções histológicas, que são especialmente evidenciadas nos órgãos ricos em elementos do sistema imunológico monocítico-fagocitário (fígado, baço, linfonodos e medula óssea). ${ }^{1,2}$ Foi classificada em três tipos (1, 2 e 3), tendo como base a presença e a gravidade de envolvimento neurológico. $\mathrm{O}$ tipo 1 ou não neuropático (adulto) é a forma mais comum da doença, correspondendo a 99\% dos casos. É especialmente prevalente entre os judeus Ashkenazi com uma incidência de cerca de 1:450 indivíduos e de 1:40.000 na população

${ }^{1}$ Professor do Departamento de Clínica Médica; Chefe do Serviço de Hemoterapia (SHMT); Chefe do Serviço de Hemovigilância;

Hospital Universitário Prof. Dr. Polydoro Ernani de São Thiago - Universidade Federal de Santa Catarina (UFSC).

${ }^{2}$ Médica do Serviço de Hemoterapia (SHMT), Hospital Universitário Prof. Dr. Polydoro Ernani de São Thiago, UFSC.

${ }^{3}$ Aluno do curso de graduação em Medicina - Faculdade Evangélica do Paraná.

Hospital Universitário Prof. Polydoro Ernani de São Thiago (HU), Universidade Federal de Santa Catarina (UFSC).

Correspondência: Vera Lúcia Paes Cavalcanti Ferreira

Universidade Federal de Santa Catarina - Centro de Ciências da Saúde

Hospital Universitário - Serviço de Hemoterapia - Campus Universitário - Trindade

88040-970 - Florianópolis-SC - Brasil

Tel.: (48) 3721-9114

E-mail:verapaz@hu.ufsc.br 
geral. O tipo 2 ou forma neuropática aguda (infantil) caracteriza-se pelo início precoce e rapidamente progressivo dos sintomas na infância (entre quatro e seis meses) e pelo acentuado comprometimento neurológico. O tipo 3 ou neuropático subagudo (juvenil) manifesta-se com distúrbios neurológicos no final da primeira década de vida e tem uma progressão mais lenta do que a forma aguda. ${ }^{3}$

O diagnóstico da DG é estabelecido por teste que demonstra a deficiência da atividade da enzima glucocerebrosidase nos leucócitos e fibroblastos da pele cultivados, com um resultado que geralmente expressa atividade $30 \%$ menor que a normal. ${ }^{3}$ Transmitida por herança autossômica recessiva, definida pela presença de dois alelos mutantes para o gene da beta-glicosidase localizado na região q21 do cromossomo 1. A presença de dois alelos mutantes deste gene serve como confirmação do diagnóstico. A biópsia de medula óssea pode demonstrar a presença de células de Gaucher, entretanto, as pseudocélulas de Gaucher podem muitas vezes levar a diagnósticos errôneos, sendo imperativo a dosagem enzimática para o diagnóstico. ${ }^{4}$

O tratamento da maioria dos pacientes sintomáticos era essencialmente de suporte clínico, envolvendo o uso de analgésicos e transfusões sangüíneas. A partir de 1990 foi possível demonstrar respostas clínicas animadoras com glucocerebrosidase derivada de placenta humana (alglucerase) e que tinha os macrófagos como alvos. ${ }^{4-6}$ Esta tinha um limite de fornecimento e foi substituída por uma forma de enzima desenvolvida a partir da técnica do DNA recombinante, a imiglucerase, capaz de suprir a produção em grandes quantidades e com excelentes resultados. ${ }^{7}$

\section{Casuística e Método}

Foram selecionados para o estudo os prontuários de pacientes com diagnóstico de doenças lisossômicas (CID-9: 272.7/8 e CID-10: E75.2) registrados no Setor de Prontuário de Pacientes (SPP) do Serviço de Arquivo Médico e Estatística (Same) do Hospital Universitário Dr. Polydoro Ernani de São Thiago (Florianópolis - SC) no período de junho de 1998 a junho de 2003. As variáveis estudadas foram: idade, gênero, raça, etnia, município de procedência, classificação quanto ao tipo da doença, ${ }^{2}$ dosagem da enzima glucocerebrosidase, ${ }^{4}$ genótipo ${ }^{8}$ (principais mutações encontradas), manifestações clínicas ${ }^{2}$ e laboratoriais antes e durante a TRE, estudos de imagem (Raios-X, ultra-sonografias abdominais e ressonância magnética).

A classificação da doença de Gaucher em tipo 1, 2 e 3 foi baseada nas caraterísticas clínicas de acordo com o início, intensidade da hepatoesplenomegalia, hiperesplenismo, manifestação óssea, envolvimento neurológico, sobrevida. ${ }^{1,2}$

Diagnóstico enzimático: foram coletados $10 \mathrm{~mL}$ de sangue periférico em seringa com $0,2 \mathrm{~mL}$ de heparina para análise da quantificação das enzimas glucocerebrosidase nos leucócitos (valores normais: 10-45 nmmol/h/mg proteína) e quitotriosidase (valores normais: 8,85-132 nmol/h/mL) no plasma. ${ }^{4}$

Diagnóstico molecular (genótipo - DNA): foram coletados $10 \mathrm{~mL}$ de sangue periférico em seringa com ácido etilenodiaminotetracético (EDTA) para análise do DNA, bem como amostras de mucosa oral que foram coletadas com swabs e condicionadas em tubos de ensaios com meios de cultura adequados.

Avaliação clínica: os pacientes foram avaliados clinicamente com aferição do peso e da altura, além das medidas das visceromegalias, usando a unidade de centímetro e a linha hemiclavicular direita para o tamanho do fígado e a linha hemiclavicular esquerda para o tamanho do baço. Esta rotina foi adotada para todos os pacientes com DG antes e durante a TRE.

Programa de Terapia de Reposição Enzimática (TRE): com o diagnóstico confirmado pela dosagem da enzima betaglicosidase, ${ }^{9}$ solicitava-se a abertura de um processo junto à Secretaria de Saúde para aquisição da medicação específica (Solicitação de Medicamentos Excepcionais - SME). A dose inicial foi de $15 \mathrm{U} / \mathrm{kg} /$ peso, em infusões endovenosas durante duas horas, com intervalo de 15 em 15 dias, sob monitoração médica. Após a infusão, os pacientes eram liberados para o domicílio.

As variáveis categóricas foram expressas em números absolutos e percentuais. O protocolo de pesquisa e o consentimento informado foram aprovados pelo Comitê de Ética em Pesquisa em Seres Humanos da Universidade Federal de Santa Catarina.

\section{Resultados}

Esta pesquisa incluiu dez pacientes com o diagnóstico de DG procedentes do estado de Santa Catarina. O tipo neuropático subagudo não foi constatado e o tipo 1 foi o mais freqüente. Houve um discreto predomínio do grupo adulto no momento do diagnóstico. Na figura 1, observa-se extenso envolvimento visceral em um menino com DG tipo 2,

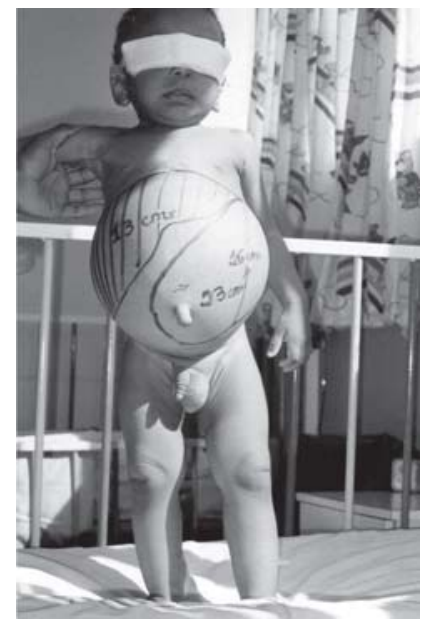

Figura 1. Caso 1 DG tipo 2 Fonte: SPP / Same - Hospital Universitário - UFSC. 
Tabela 1. Exames complementares mais freqüentemente utilizados para a investigação diagnóstica da doença de Gaucher. HU, 1998/2003

\begin{tabular}{|c|c|c|c|c|c|c|c|}
\hline Caso & $\begin{array}{c}\text { Dosagem } \\
\text { glucocerebrosidase }\end{array}$ & $\begin{array}{c}\text { Dosagem } \\
\text { Quitotriosidase }\end{array}$ & Mielograma & $\begin{array}{c}\text { Biópsia } \\
\text { Medula Óssea }\end{array}$ & $\begin{array}{c}\text { Biópsia } \\
\text { Hepática } \\
\end{array}$ & $\begin{array}{c}\text { Biópsia } \\
\text { Linfonodo }\end{array}$ & $\begin{array}{c}\text { Biópsia } \\
\text { Baço }\end{array}$ \\
\hline 1 & $(+)$ & & $(+)$ & $(+)$ & & & \\
\hline 2 & $(+)$ & & & $(+)$ & & & \\
\hline 3 & $(+)$ & $(+)$ & $(+)$ & & $(+)$ & $(+)$ & \\
\hline 4 & $(+)$ & $(+)$ & $(+)$ & & & & \\
\hline 5 & $(+)$ & $(+)$ & & & & & \\
\hline 6 & $(+)$ & & $(+)$ & $(+)$ & & & \\
\hline 7 & $(+)$ & $(+)$ & & & & & $(+)$ \\
\hline 8 & $(+)$ & & $(+)$ & $(+)$ & & & \\
\hline 9 & $(+)$ & $(+)$ & $(+)$ & & & & \\
\hline 10 & $(+)$ & $(+)$ & & $(+)$ & & & \\
\hline $\mathrm{n}$ & 10 & 6 & 6 & 5 & 1 & 1 & 1 \\
\hline$\%$ & 100 & 60 & 60 & 50 & 10 & 10 & 10 \\
\hline
\end{tabular}

Fonte: SPP / Same - Hospital Universitário - UFSC

Tabela 2. Distribuição dos pacientes com DG segundo classificação clínica e dosagem enzimática. HU, 1998/2003

\begin{tabular}{ccc}
\hline N Caso & Classificação clínica & $\begin{array}{c}\text { Glucocerebrosidase } \\
\text { nmol/h/mg proteína }\end{array}$ \\
\hline 1 & Tipo 2 & 2,12 \\
2 & Tipo 2 & 1,7 \\
3 & Tipo 1 & 0,7 \\
4 & Tipo 1 & 1,1 \\
5 & Tipo 1 & 0,2 \\
6 & Tipo 1 & 0,4 \\
7 & Tipo 1 & 0,23 \\
8 & Tipo 1 & 0,7 \\
9 & Tipo 1 & 0,96 \\
10 & Tipo 1 & 0,35 \\
\hline
\end{tabular}

Fonte: SPP / Same - Hospital Universitário - UFSC

hepatoesplenomegalia volumosa e ascite, circulação portal, caracterizando hipertensão portal. O paciente apresentou também hérnia umbilical e inguinoescrotal, secundárias à síndrome da ocupação abdominal.

A maioria dos pacientes teve o diagnóstico na fase adulta, com um leve predomínio do gênero masculino, e apenas um paciente era negro. Todos os pacientes tiveram a confirmação diagnóstica da DG pela dosagem da atividade da enzima glucocerebrosidase (Tabela 1). Os dois casos classificados como tipo 2 tiveram valores relativamente maiores da atividade da enzima glucocerebrosidase quando comparados com os demais, diagnosticados como tipo 1 (Tabela 2).

Hepatoesplenomegalia foi a manifestação clínica mais comum dos pacientes com DG. O envolvimento ósseo esteve presente em $75 \%$ dos pacientes com a forma clínica não neuropática. Osteopenia e necrose avascular foram as alterações radiológicas mais freqüentes (Tabela 3).

Anemia e trombocitopenia foram observadas em 100\% dos pacientes e $30 \%$ deles apresentaram pancitopenia.A his-
Tabela 3. Envolvimento ósseo dos pacientes com DG. HU, 1998/2003

\begin{tabular}{cll}
\hline Alterações de imagem & $\mathrm{n}$ & $\%^{*}$ \\
\hline Ausente & 4 & 40 \\
Osteopenia & 4 & 40 \\
Necrose avascular & 3 & 30 \\
Deformidade em frasco de Erlenmeyer & 2 & 20 \\
Infarto ósseo & 1 & 10 \\
Infiltração da medula & 1 & 10 \\
Fratura & 1 & 10 \\
\hline
\end{tabular}

*Percentual calculado em relação ao total de pacientes $(n=10)$ Fonte: SPP / Same - Hospital Universitário - UFSC

tória clínica envolvendo outros membros da família com DG esteve presente em $40 \%$ dos casos. Todos os pacientes com a apresentação clínica tipo 1 e com história familiar apresentaram o alelo mutante N370S, enquanto o que foi diagnosticado como tipo severo (neuropática aguda) apresentou genótipo homozigoto para a mutação L444P (Tabela 4).

O alelo N370S foi identificado em $60 \%$ dos casos (casos 4, 6, 7, 8, 9 e 10), sendo revelado em homozigose apenas no caso 9 e nos demais em heterozigose. O alelo mutante L444P esteve presente em homozigose em $10 \%$ dos casos e em heterozigose associado ao N370S em $20 \%$ dos pacientes. (Figura 2).

Tabela 4. Distribuição dos pacientes com história familiar de DG de acordo com a classificação clínica e genótipo. HU, 1998/2003

\begin{tabular}{cccc}
\hline $\begin{array}{c}\text { Classificação da } \\
\text { doença }\end{array}$ & $\mathrm{n}$ & \%* & Genótipo \\
\hline Tipo 1 & 3 & 75 & N370S/? \\
Tipo 2 & 1 & 25 & L444P/L444P \\
\hline Total & 4 & 100 & \\
\hline
\end{tabular}

*Percentual calculado em relação ao $\mathrm{n}^{0}$ de pacientes com história familiar $(n=4)$

Fonte: SPP/Same - Hospital Universitário - UFSC 


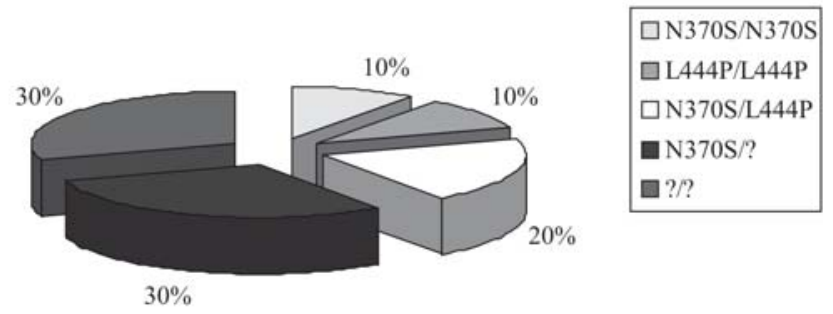

Figura 2 . Distribuição dos pacientes com DG segundo a ocorrência dos alelos mutantes no gene da glucocerebrosidase. HU, 1998/ 2003. Fonte: SPP/Same - Hospital Universitário - UFSC
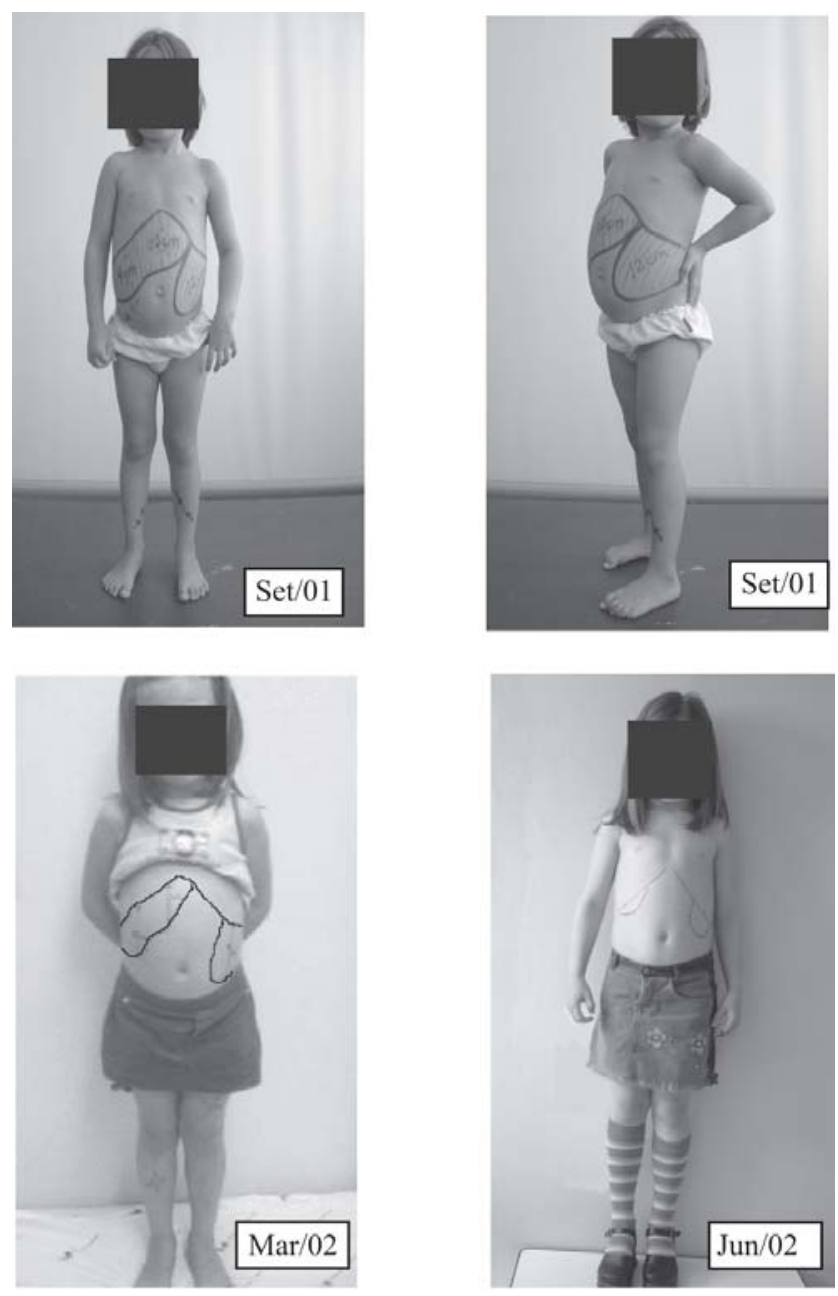

A figura 3 mostra o tamanho inicial da hepatoesplenomegalia e a redução da mesma, após nove meses de Terapia de Reposição Enzimática, em uma menina com doença de Gaucher tipo1.

\section{Discussão}

A DG permaneceu durante o século XX, praticamente, sem opção terapêutica específica e rompe o novo milênio com a descoberta de um tratamento efetivo e seguro. Tornou-se um modelo genético para a implementação de testes diagnósticos pré-natais, correlação genótipo/fenótipo e foi a primeira deficiência enzimática intracelular a ser tratada com uma enzima produzida por método recombinante. ${ }^{7}$

Esta pesquisa reuniu dez casos de DG procedentes de diversos municípios de Santa Catarina, devidamente estudados, diagnosticados e acompanhados no Hospital Universitário Dr. Polydoro Ernani de São Thiago, da Universidade Federal de Santa Catarina.

Em um estudo multicêntrico, com a participação de vários países e com uma casuística significante (1.698 pacientes - The Gaucher Registry), constatou-se uma freqüência de $94 \%$ de pacientes com o tipo 1 , menos de $1 \%$ de pacientes do tipo 2 e $5 \%$ com o tipo $3 .{ }^{8}$ Este estudo detectou oito casos de DG tipo 1 e dois casos do tipo 2. A casuística mostrou uma frequêencia de $80 \%$ de indivíduos com a DG tipo 1, concordando com a literatura. O tipo 2 esteve presente em dois casos (20\%) e não houve registro do tipo 3.

A DG tipo 2 (Figura 1) é a mais rara (1/100.000 a 1/ 500.000 nascimentos), a mais grave forma da doença e foi reconhecida em 1972 como um fenótipo distinto, geralmente progressiva e fatal, ocasionando a morte precocemente. ${ }^{3}$ Os dois pacientes que apresentaram este tipo de DG manifestaram envolvimento extenso visceral, caracterizado por hepatoesplenomegalia volumosa e grave trombocitopenia. Esses pacientes não apresentaram resposta à TRE e foram ao óbito aos dois anos de idade.

O diagnóstico de DG deve ser evocado nos casos de esplenomegalia inexplicada, com ou sem história de diásteses hemorrágicas ou outras manifestações esqueléticas, bem como nos casos de crianças com hepatoesplenomegalia associada a um quadro clínico neurodegenerativo. ${ }^{1,2}$ A presença de células de Gaucher em aspirados de medula óssea é altamente sugestiva da doença. Entretanto, muitas células semelhantes (pseudocélulas de Gaucher) têm sido descritas numa variedade de doenças, inclusive na síndrome da imunodeficiência adquirida (SIDA) e nas infecções por Mycobacterium avium, conduzindo a diagnósticos errôneos. ${ }^{2}$ Os exames que permitiram o diagnóstico da DG neste estudo foram: mielograma, biópsia de medula óssea, biópsia hepática, biópsia de linfonodo, biópsia esplênica, dosagem da atividade das enzimas: quitotriosidase e glicocerebrosidase. Entre os exames passíveis de realização em Santa Catarina, o mielograma e a biópsia de medula óssea foram os mais comuns, com um predomínio de $60 \%$ e $50 \%$, respectivamente (Tabela 1).

Para estabelecer o diagnóstico definitivo, de maneira eficiente e confiável, deve-se solicitar a dosagem da atividade da enzima glucocerebrosidase, que pode ser aferida nos leucócitos do sangue periférico ou fibroblastos cultivados. ${ }^{2,48}$ Apesar do teste enzimático confirmar o diagnóstico com uma leitura menor que 30\% da atividade enzimática normal, este não diferencia as variantes clínicas. ${ }^{2}$ Em todos os pacientes, a atividade da enzima foi decisivamente deficiente e o principal critério de inclusão dos casos (Tabela 2). 
Quanto às manifestações clínicas, sabe-se que a esplenomegalia está geralmente presente nos pacientes com DG. O aumento do volume hepático também é regra, chegando a preencher o abdome por completo nas formas graves. ${ }^{2,10}$ A figura 1 mostra extenso envolvimento visceral, com ocupação completa da cavidade abdominal, em um lactente com a variante clínica tipo 2.

A hepatoesplenomegalia foi o mais freqüente sinal patológico encontrado no exame físico dos pacientes estudados, tendo sido observado em $80 \%$ dos casos, porém a investigação ultra-sonográfica abdominal revelou aumento de baço e fígado em $100 \%$. Em dois casos, a esplenomegalia não foi evidenciada ao exame clínico, sendo que o diagnóstico foi realizado na fase adulta e as principais manifestações clínicas foram ósseas, com alterações radiológicas significativas e graves, inclusive com prejuízo das atividades funcionais.

As manifestações esqueléticas são definidas como efeitos secundários da deficiência da beta-glicocerebrosidase sob a mineralização óssea, que muitas vezes não são devidamente reconhecidas e podem ser o aspecto mais debilitante da DG. ${ }^{2,11,12} \mathrm{O}$ envolvimento ósseo, quando investigado através de radiografias, cintilografias, tomografias computadorizadas ou imagens de ressonância magnética, está presente na maioria dos pacientes afetados pela doença. ${ }^{13-17}$ (Tabela 3)

A anemia é geralmente leve, mas ocasionalmente intensa com hemoglobina abaixo de $5 \mathrm{~g} / \mathrm{dL}$. Leucopenia e pancitopenia também ocorrem em alguns pacientes. Estas alterações são muito provavelmente secundárias a uma combinação de seqüestração esplênica pela esplenomegalia e hipofunção medular devido à substituição da medula óssea pelas células de Gaucher. ${ }^{2}$ As alterações hematológicas mais freqüentemente encontradas nesta casuística foram: anemia (100\%), plaquetopenia (100\%), leucopenia (50\%), neutropenia (50\%) e pancitopenia (30\%).

A pesquisa das mutações mais freqüentes para o gene da glucocerebrosidase dos pacientes com história familiar de DG demonstrou a presença dos alelos N370S e L444P (Tabela 4).

O alelo N370S esteve presente em 60\% dos casos, sendo que em $50 \%$ os indivíduos tinham genótipos compostos heterozigotos e os $10 \%$ restantes homozigotos. A mutação mais freqüente foi representada pelo genótipo N370S/? (30\%), seguida do heterozigoto N370S/L444P (20\%). (Figura 2).

A primeira mutação descrita em uma criança com DG tipo 2 foi a L444P e continua sendo a mutação mais freqüentemente encontrada neste grupo de pacientes. ${ }^{18-20} \mathrm{~A}$ análise de DNA do caso 1 revelou genótipo L444P/L444P (Figura 1) e o estudo biomolecular (DNA) dos pais demonstrou que ambos eram heterozigotos para esta mutação. Este paciente apresentava rica história familiar, os pais eram primos (consangüíneos) e tinham perdido uma filha aos seis meses de idade, em um outro hospital, com quadro clínico semelhante, após uma esplenectomia precoce.
Torna-se essencial o estudo do genótipo como instrumento básico para o aconselhamento genético dos afetados e portadores da DG, com a finalidade de reduzir os riscos potenciais.

Em contraste com outras doenças genéticas, para a DG tipo 1 existe um tratamento altamente efetivo. ${ }^{8}$ A terapia de reposição enzimática (TRE) tem mudado significativamente a evolução desta apresentação clínica, permitindo benefícios na qualidade de vida destes pacientes, interrompendo, diminuindo ou normalizando com segurança e eficácia muitos de seus maiores sinais e sintomas. ${ }^{21,22}$

A análise da resposta à TRE de 33 pacientes com DG tipo 1, nos Estados Unidos da América, demonstrou um acréscimo médio nos níveis de hemoglobina de $1,3 \mathrm{~g} / \mathrm{dL}$ após seis meses de tratamento com reposição enzimática em 18 pacientes não esplenectomizados. ${ }^{23}$ Ao término deste estudo, oito pacientes com DG tipo 1 encontravam-se clinicamente estáveis, em TRE, na dependência do fornecimento da enzima. Apesar das infusões ocorrerem de forma irregular devido à dificuldade na aquisição da medicação, do período de tratamento ter variado entre quatro a 36 meses, esta pesquisa demonstrou que, de um modo geral, a resposta clínica foi significativa, traduzida pela elevação da taxa de hemoglobina, com um aumento médio de 1,2 g/dL, além da redução da hepatomegalia e da esplenomegalia com retomada do crescimento dos casos pediátricos de acordo com o potencial genético (Figura 3). Não foram observados efeitos adversos durante o acompanhamento destes pacientes.

Algumas limitações foram observadas durante esta pesquisa: impedimento de uma avaliação mais rigorosa da TRE em virtude das interrupções do regime quinzenal das infusões da imiglucerase pela falta do medicamento, dificuldade de se estabelecer o melhor esquema de dose terapêutica da reposição enzimática devido ao grau de variabilidade da gravidade da doença e à impossibilidade de se avaliar a resposta das manifestações esqueléticas, que ficou prejudicada em virtude dos seguintes fatores: curto intervalo de tempo da TRE, dificuldade de se elevarem as doses para $60 \mathrm{U} / \mathrm{kg}$ de peso e de se realizar a ressonância magnética.

Devido à heterogeneidade e à natureza sistêmica da DG, a posologia da terapia de reposição enzimática deve ser individualizada, tendo como base critérios clínicos, radiológicos e laboratoriais. Há consenso internacional de que a DG assintomática não é por si só indicação da terapia de reposição enzimática. ${ }^{24}$ A Secretaria de Assistência à Saúde, pela portaria $n^{\circ} 449$ de 09 de julho de 2002, estabeleceu um Protocolo Clínico e Diretrizes Terapêuticas para o tratamento da DG e para a liberação do medicamento imiglucerase. ${ }^{9}$

No mundo, atualmente mais de 3 mil pacientes com este tipo de enfermidade estão recebendo infusões com glucocerebrosidase recombinante (imiglucerase). A maioria dos pacientes tratados apresenta uma reversão dos sintomas ou bloqueio da doença. Entretanto, a viabilidade de um trata- 
mento totalmente efetivo com a proposta de cura para a DG será possível somente com terapia gênica. ${ }^{25}$

A DG é considerada rara, pouco conhecida no meio médico nacional, apesar do avanço técnico-científico das últimas décadas. A relevância dessa pesquisa é difundir os novos conhecimentos sobre a doença de Gaucher para se estabelecer um diagnóstico o mais precocemente possível e conseqüentemente promover uma melhor qualidade de vida para estes pacientes.

\section{Conclusões}

1. A doença de Gaucher tipo 1 é a forma clínica mais comum. Anemia, trombocitopenia, hepatoesplenomegalia e osteopenia, associadas à deficiência enzimática da glicocerebrosidase, são as características mais freqüentes dos pacientes com doença de Gaucher em Santa Catarina.

2. O alelo N370S é o mais freqüente e está relacionado com o tipo 1. O alelo L444P em homozigose sugere letalidade precoce. O estudo do genótipo auxilia no suporte clínico e é necessário para o aconselhamento genético.

3. ATRE é efetiva e segura no tratamento da doença de Gaucher tipo l.

\begin{abstract}
Gaucher Disease (GD) was the first described and is the most common lysosomal deposit disease. It is characterized byahereditary deficiency of glucocerebrosidase lysosomal enzyme activity which blocks the metabolism of glucocerebrosideo. The aim of this work was to study the clinical, laboratorial and radiological characteristics, the main mutations correlating them with the clinical form of the disease and evaluating the response to enzymatic replacement therapy in patients with GD in Santa Catarina. Ten GD patients were studied at a University Hospital between 1998 and 2003. The disease was diagnosed by measurement of the betaglucosidase enzyme in leukocytes. Investigation of mutations used samples of blood and oral mucus. The average age at diagnosis was 19.6 years. Type 1 GD was diagnosis in $80 \%$ of the cases and type 2 in 20\%. Four patients had a family history of GD. Hepatosplenomegaly was the most common clinical manifestation. Anemia and thrombocitopenia occurred in all cases. Bone pain was reported by $75 \%$ of the patients. The mutanted alleles identified were N370S and L444P. The hemoglobina levels were elevated in all patients with type $1 \mathrm{GD}$. In conclusion, type $1 \mathrm{GD}$ is the most common clinical form and anemia, thrombocitopenia, hepatosplenomegaly and osteopenia were the most common characteristics of GD patients. The N370S allele is the most common mutation and is related with type 1 GD 1 . Homozygosity of the L444P allele suggests early death. Enzyme replacement therapy is safe and efficacious in type $1 \mathrm{GD}$. Rev. bras. hematol. hemoter. 2008;30(1):5-11.
\end{abstract}

Key words: Gaucher disease; lipidosis; glucocerebrosidase; genotype.

\section{Agradecimentos}

Aos pacientes com doença de Gaucher e familiares que, no anonimato, foram os protagonistas e a motivação permanente deste estudo.

Aos pesquisadores Janice Carneiro Coelho, Maria Luíza Pereira, Kristiane Michelin (Universidade Federal do Rio Grande do Sul) e Roberto Rozenberg (Universidade de São Paulo), pela colaboração essencial na investigação laboratorial destes pacientes.

Aos funcionários dos Serviços de Pediatria, Hematologia e Hemoterapia do Hospital Universitário da Universidade Federal de Santa Catarina.

\section{Referências Bibliográficas}

1. Nathan DG, Oski FA. Storage diseases of the reticuloendotelial system: Gaucher's disease. In: Nathan DG, Oski FA, editors. Nathan and Oski's Hematology of Infancy and Childhood. 5th ed. Philadelphia: WB Saunders; 1998. p.1474-8.

2. Beautler E, Grabowski GA. Gaucher disease. In: Scriver CR, Beaudet AL, Sly WS, Valle D, editors. The metabolic and molecular bases of inherited disease. 8th Ed. New York: McGraw-Hill; 2001.p.3635-68.

3. Grabowski GA. Gaucher disease: enzymology, genetics, and treatment. In: Harris H, Hirschhorn K, editors. Advances in human genetics. New York: Plenum Press; 1993. p.377-441.

4. NIH Technology Assesment Panel on Gaucher Disease. Gaucher disease: current issues in diagnosis and treatment. JAMA. 1996; 275(7):548-53.

5. Mistry PK, Wraight EP, Cox TM. Therapeutic delivery of proteins to macrophages: implications for treatment of Gaucher's disease. Lancet. 1996;348:1555-9.

6. Barton NW, Furbish FS, Murray GJ, Garfield M, Brady RO. Therapeutic response to intravenous infusions of glucocerebrosidase in a patient with Gaucher disease. Proc Natl Acad Sci USA. 1990;87:1913-6.

7. Grabowski GA, Barton NW, Pastores G, Dambrosia JM, Banerjee TK, McKee MA et al. Enzyme therapy in type 1 Gaucher disease: comparative efficacy of mannose-terminated glucocerebrosidase from natural and recombinant sources. Ann Intern Med. 1995; 122:33-9.

8. Charrow J, Anderson HC, Kaplan P, Kolodny EH, Mistry P, Pastores $\mathrm{G}$ et al. The Gaucher registry: demographics and disease characteristics of 1698 patients with Gaucher disease. Arch Intern Med. 2000;160(18):2835-43.

9. Brasil. Ministério da Saúde. Portaria n 449 de 09 de julho de 2002. Protocolo clínico e diretrizes terapêuticas - doença de Gaucher imiglucerase. 2002.09.11. Available from: URL: http:/www.saúde. gov.br/sas/portarias/port2002/pt-449.htm.

10. Balicki D, Beutler E. Gaucher disease: reviews in molecular medicine. Medicine. 1995;74(6):305-23.

11. Pastores GM, Einhorn TA. Skeletal complications of Gaucher disease: pathology, evaluation and treatment. Semin Hematol 1995; 32(7):20.

12. Stowens DW, Teitelbaum SL, Kahn AJ, Barranger JA. Skeletal complications in Gaucher disease. Medicine. Baltimore: 1985;64:310.

13. Hermann G, Pastores GM, Abdelwahab IF, Loberboym AM. Gaucher disease: assessment of skeletal involvement and therapeutic responses to enzyme replacement. Skeletal Radiol. 1997;12:687-96.

14. Rosenthal DI, Scott JA, Barranger J, Mankin HJ, Saini S, Brady TJ et al. Evaluation of Gaucher disease using magnetic resonance imaging. J Bone Joint Surg Am. 1986;68:802. 
15. Cremim BJ, Davey H, Goldblatt J. Skeletal complications of type I Gaucher disease: the magnetic resonance features. Clin Radiol. 1990;41:244-7.

16. Rosenthal DI, Barton NW, McKusick KA, Rosen BR, Hill SC, Castronovo FP et al. Quantitative imaging of Gaucher disease. Radiology. 1992;185:841.

17. Rosenthal DI, Mayo-Smith W, Goodsitt MM, Doppelt S, Mankin HJ. Bone and bone marrow changes in Gaucher disease: evaluation with quantitative CT. Radiology. 1989;170:143.

18. Weinreb, NJ. Type 1 Gaucher disease in elderly patients. Gaucher Clin Perspect. 1999;7(2):1-8.

19. Tayebi N, Stone D, Sidransky E. Type 2 Gaucher disease: an expanding phenotype [minireview]. Mol Genet Metabol. 1999;68: 209-19.

20. Sidransky E, Fartasch M, Lee RE, Metlay LA, Abella S, Zimran A et al. Epidermal abnormalities may distinguish type 2 from type 1 and type 3 of Gaucher disease. Pediatr Res. 1996;39:134.

21. Barton NW, Brady RO, Dambrosia JM. Replacement therapy for inherited enzyme deficiency - macrophage-targeted glucocerebrosidase for Gaucher's disease. N Engl J Med. 1991;324:1464-70.

22. Pastores G. Recombinant enzyme therapy for Gaucher disease: longterm clinical experience and therapeutic outcomes with crossover from alglucerase. Gaucher Clin Perspect. 1998;6(1):5-8.

23. Pastores GM, Sibille AR, Grabowski GA. Enzyme therapy in Gaucher disease type 1: dosage efficacy and adverse effects in 33 patients treated for 6 to 24 months. Blood. 1993;82:408-16.

24. Beutler E, Demina A, Laubscher K, Garver P, Gelbart T, Balicki D et al. The clinical course of treated and untreated Gaucher disease. A study of 45 patients. Blood Cells Mol Dis. 1995;21(10):86-108.

25. Cox T, Lachman R, Hollak C. Novel oral treatment of Gaucher's disease with N-butydeoxynojirimycin (OGT 918) to decrease substrate biosynthesis. Lancet. 2000;355(9214):1481-5.

Avaliação: Editor e dois revisores externos

Conflito de interesse: sem conflito de interesses.

Recebido: 02/05/06

Aceito após modificações: 27/12/06 